\title{
碱性蛋白酶催化合成喹唑啉酮衍生物
}

\author{
谢宗波* 李红霞刘联胜兰金故宇乐长高* \\ (东华理工大学应用化学系 南昌 330013)
}

\begin{abstract}
摘要 以碱性蛋白酶为生物催化剂, 通过二羰基化合物和邻氨基苯甲酰胺间的缩合反应合成了喹唑啉酮类衍生物. 乙 醇作为环保型溶剂，可以减少反应对环境的影响. 碱性蛋白酶具有催化活性高，环保，来源广泛，操作简单等优点. 本 方法仅使用 $2000 \mathrm{U}$ 碱性蛋白酶作为催化剂, 即可获得各种喹唑啉酮衍生物, 且具有良好至优异的产率.

关键词＼cjkstart喹唑啉酮衍生物; 生物催化; 碱性蛋白酶; 乙醇; 非专一性
\end{abstract}

\section{Synthesis of Quinazolinone Derivatives Catalyzed by Alkaline Protease}

\author{
Xie, Zongbo* Li, Hongxia $\quad$ Liu, Liansheng Lan, Jin $\quad \mathrm{Hu}$, Zhiyu $\quad$ Le, Zhanggao* \\ (Department of Applied Chemistry, East China University of Technology, Nanchang 330013)
}

\begin{abstract}
Alkaline protease-catalyzed synthesis of quinazolinone derivatives was developed between $\beta$-keotester and $o$-aminobenzamide. Because ethanol is one kind of eco-friendly solvents, this method can reduce the impact of solvents on the environment. Alkaline protease as a biocatalyst has many advantages, e.g. high catalytic activity, environmentally friendly, wide variety of sources and simple operation. In addition, a variety of quinazolinone derivatives was obtained with good to excellent yields just using $2000 \mathrm{U}$ alkaline protease as catalyst.
\end{abstract}

Keywords quinazolinone derivatives; biocatalysis; alkaline protease; ethanol; promiscuity

\section{Introduction}

Quinazolinone derivatives are abundant in a broad number of pharmaceutical compounds, and are widely applied as sedative, hypotensive, antidepressant, anti-inflammatory as well as anti-allergy activities owing to its promising bioactivities. ${ }^{[14]}$ Therefore, tremendous efforts have been devoted to develop the methodology to construct quinazolinone derivatives during past decades. Conventional protocols were intensively focused on the condensation of $o$-aminobenzamide with carbonyl compounds using Lewis acids as catalyst, in which transition-metal and long reaction time were required to obtain the satisfactory yields or under harsh conditions. ${ }^{[5 \sim 14]}$ With the gradual development of organic synthesis, reductive cyclization of the aldehyde with anthranilic acid nitrile has also been developed. ${ }^{[15 \sim 17]}$ Especially, quinazolinone derivatives were also readily accessed by acetic acid-catalyzed coupling of benzylamine/aromatic alcohol with $o$-aminobenzamide. ${ }^{[18 \sim 19]}$
In addition, synthesis of quinazolinone derivatives from ammonium acetate, homophthalic anhydride and aromatic aldehyde without catalyst has been developed. ${ }^{[20]}$ Although significant achievements have been made, there are still several drawbacks in the reported methodologies, including harsh reaction conditions, long reaction time, strongly acidic conditions, high loading of expensive catalysts (additives), and difficulty in experiment handling. Therefore, effective, eco-friendly and sustainable biocatalytic methods are still in high demand.

Compared with chemical catalysts, biocatalysts have attracted many attentions owing to low toxicity, high efficiency, and good selectivity, such as chemical, regional, and optical selectivities, which is resulting in numerous applications in the agrochemical products, drugs, and refined chemicals. ${ }^{[21 \sim 23]}$ As a biocatalyst, enzymes are ubiquitous in animals, plants and microorganisms, and can be directly isolated and purified from organisms. The definition of the "unit of enzyme activity" (symbol U) is the

\footnotetext{
* Corresponding authors. E-mail: zbxie@ecit.cn; zhgle@ecit.cn

Received January 24, 2019; revised March 27, 2019; published online April 11, 2019.

Project supported by the National Natural Science Foundation of China (Nos. 21462001, 21465002, 21262002), the Science and Technology Projects of Jiangxi (No. 20161BCB24006), and the Science and Technology Foundation of the Jiangxi Education Department (Nos. KJLD14050, GJJ150584).

国家自然科学基金(Nos. 21462001, 21465002, 21262002)、江西省科技计划(No. 20161BCB24006)、江西省教育厅科技(Nos. KJLD14050, GJJ150584)资 助项目.
} 
amount of enzyme which catalyzes the transformation of one micromole of substrate per minute under standard assay conditions. ${ }^{[24]}$ As efficient biocatalysts, enzymes help to carry out multi-functional non-natural reactions, ${ }^{[25]}$ because of their catalytic promiscuity, which strongly facilitate their application in the realm of organic synthesis, such as aldol reactions, ${ }^{[26,27]}$ Michael reactions, ${ }^{[28-31]}$ Mannich reactions ${ }^{[32,33]}$ and Henry reactions. ${ }^{[34,35]}$ As a kind of protease, alkaline protease exhibits higher catalytic activities and selectivities in synthesis of heterocyclic compounds. ${ }^{[36 \sim 38]}$ In order to extend the application of enzyme in more challenging organic synthesis, we envisioned that alkaline protease catalysis may provide a practical approach to directly accessing various functional quinazolinone derivatives.

Here, alkaline protease was selected as the biocatalyst, and a series of quinazolinone derivatives can readily be obtained from the substituted o-aminobenzamide and $\beta$-ketoester under the mild reaction conditions. In contrast to previous reports, our protocol provides a metal-free approach to various functional-group quinazolinone derivatives in excellent yields in ethanol (Scheme 1).

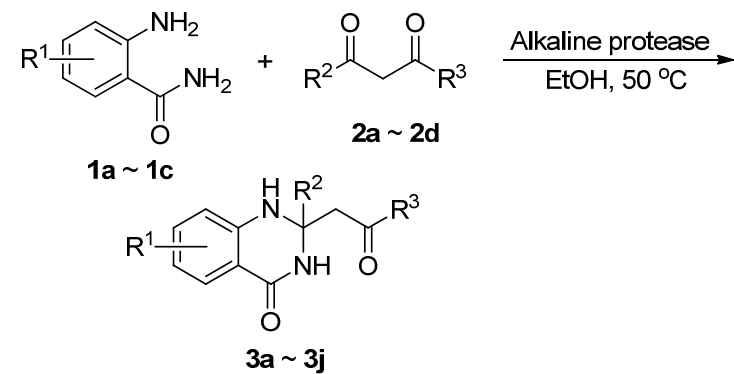

Scheme 1 Alkaline protease-catalyzed synthesis of quinazolinone derivatives

\section{Results and discussion}

To confirm our hypothesis, the catalytic activities of various enzymes were explored using $o$-aminobenzamide 1a and ethyl acetoacetate $\mathbf{2 a}$ as a model reaction (Table 1). No product was observed in the absence of enzyme (Table 1, Entry 19). After a detailed optimization of various reaction conditions, 91\% yield of the corresponding quinazolinone derivatives $\mathbf{3 a}$ was obtained in the presence of alkaline protease and ethanol as solvent at $50{ }^{\circ} \mathrm{C}$ for $3 \mathrm{~d}$ (Table 1, Entry 1). At the same time, yields $46 \%$ and $23 \%$ of the corresponding quinazolinone derivative $\mathbf{3 a}$ were obtained using pancreatin and trypsin as catalysts, respectively (Table 1, Entries 2 3). When other enzymes were applied under standard conditions, the product was not observed (Table 1, Entries 4 18). No product was detected when the reaction was incubated with denatured alkaline proteinase, which suggested that the specific structure of enzyme was necessary to carry out this biocatalytic reaction (Table 1, Entry 20).

Solvent is an important factor affecting the reaction effect, and the effect of solvent was studied under the catalytic of alkaline protease. As can be seen from Table 2,
Table 1 Catalytic activities of different enzymes ${ }^{a}$

\begin{tabular}{|c|c|c|}
\hline Entry & Enzyme & Yield $^{b} / \%$ \\
\hline 1 & Alkaline protease $(200 \mathrm{U} / \mathrm{mg})$ & 91 \\
\hline 2 & Pancreatin (4 U/mg) & 46 \\
\hline 3 & Trypsin (2500 U/mg) & 23 \\
\hline 4 & Pancreatic lipase (30-90 U/mg) & None \\
\hline 5 & Albumin from Bovine serum & None \\
\hline 6 & Lipase $(30 \sim 90 \mathrm{U} / \mathrm{mg})$ & None \\
\hline 7 & $\alpha$-Chymotrypsin (800 U/mg) & None \\
\hline 8 & Pepsin from Porcine gastric mucosa $(601 \mathrm{U} / \mathrm{mg})$ & None \\
\hline 9 & Protease from Aspergillus melleus (3.3 U/mg) & None \\
\hline 10 & Papain from Papayalatex (800 U/mg) & None \\
\hline 11 & $\begin{array}{l}\text { Amano lipase A from Aspergillus niger ( } 300 \\
\mathrm{U} / \mathrm{mg})\end{array}$ & None \\
\hline 12 & $\begin{array}{l}\text { Amano lipase ps from Burkholderia Cepacia } \\
(30 \mathrm{U} / \mathrm{mg})\end{array}$ & None \\
\hline 13 & Protease from Aspergillus saitoi $(15 \sim 35 \mathrm{U} / \mathrm{mg})$ & None \\
\hline 14 & Glucanase (2 U/mg) & None \\
\hline 15 & $\begin{array}{l}\text { Amano lipase from Pseudomonas fluorescens } \\
(20 \mathrm{U} / \mathrm{mg})\end{array}$ & None \\
\hline 16 & $\alpha$-Amylase $(10 \mathrm{U} / \mathrm{mg})$ & None \\
\hline 17 & Lipase from Porcine pancreas (100 U/mg) & None \\
\hline 18 & Amano acylase $(30 \mathrm{U} / \mathrm{mg})$ & None \\
\hline 19 & No enzyme & None \\
\hline 20 & Denatured alkaline protease $^{c}$ & None \\
\hline
\end{tabular}

the highest yield was obtained in ethanol compared to other solvents. The yield of this reaction in protic solvents is better than that in aprotic solvents, the reason may be that it is more conducive to electron conduction and energy transmission in protic solvents, which is favorable for the reaction.

Table 2 Effect of solvent on reaction ${ }^{a}$

\begin{tabular}{clc}
\hline Entry & Solvent & Yield $^{b} \%$ \\
\hline 1 & $\mathrm{H}_{2} \mathrm{O}$ & 61 \\
2 & Ethanol & 91 \\
3 & Toluene & 0 \\
4 & THF & 11 \\
5 & Acetonitrile & 27 \\
\hline
\end{tabular}

${ }^{a}$ Reaction conditions: $o$-aminobenzamide $(0.2 \mathrm{mmol})$, ethyl acetoacetate $(0.2$ $\mathrm{mmol})$, alkaline protease $(4000 \mathrm{U})$, solvent $(2 \mathrm{~mL}), 50{ }^{\circ} \mathrm{C}, 3 \mathrm{~d} .{ }^{b}$ Yield of isolated compound.

Due to the key influencing factors in most biocatalytic reactions, the effect of temperature was studied under the catalytic of alkaline proteinase. As can be seen from Figure $1,91 \%$ yield was obtained at $50{ }^{\circ} \mathrm{C}$ in $3 \mathrm{~d}$. Though a higher yield was obtained at $60{ }^{\circ} \mathrm{C}, 50{ }^{\circ} \mathrm{C}$ was chosen as the optimal temperature in terms of the energy consumption and the inactivation of enzyme at high temperature for a long time.

Then, the effect of the amount of catalyst on the reaction was investigated. The results are shown in Figure 2. As the amount of enzyme increased, the yield gradually increased, 


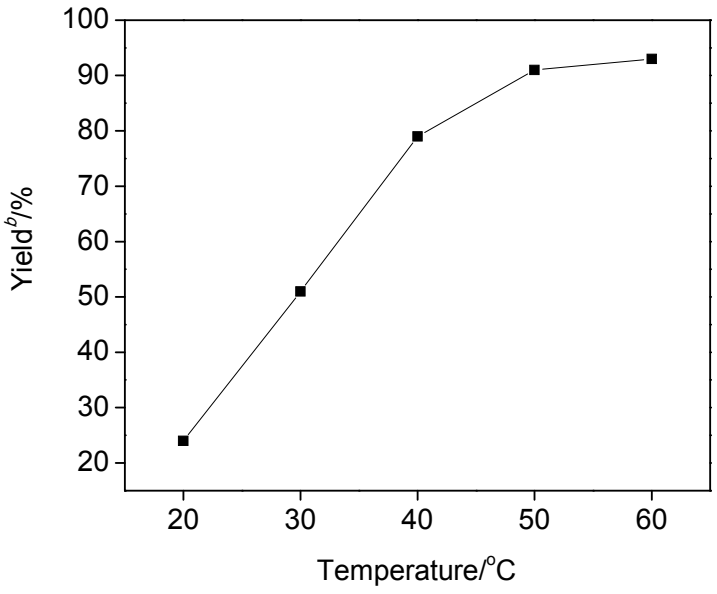

Figure 1 Influence of temperature on the model reaction Reaction conditions: $o$-aminobenzamide $(0.2 \mathrm{mmol})$, ethyl acetoacetate $(0.2 \mathrm{mmol})$, alkaline protease $(4000 \mathrm{U})$, ethanol $(2 \mathrm{~mL}), 3 \mathrm{~d}$. Yield of isolated compound

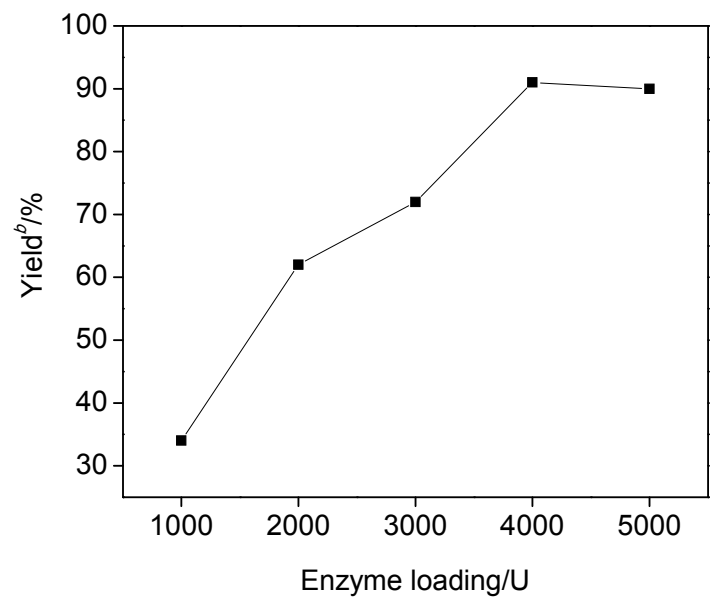

Figure 2 Effect of enzyme loading on the yield of reaction Reaction conditions: $o$-aminobenzamide $(0.2 \mathrm{mmol})$, ethyl acetoacetate $(0.2 \mathrm{mmol})$, alkaline protease $(1000 \sim 5000 \mathrm{U})$, ethanol $(2 \mathrm{~mL}), 50^{\circ} \mathrm{C}, 3$ d. Yield of isolated compound

and when the amount of the enzyme was increased to 4000 $\mathrm{U}$, the yield was $91 \%$. With the further increase in the amount of nzyme, the yield did not increase significantly. Therefore, considering the efficiency of the reaction and the principle of green chemistry, the final choice of $4000 \mathrm{U}$ is the optimal catalyst dosage.

As shown in Figure 3, as the reaction time increases, the yield gradually increases. When the reaction time was $3 \mathrm{~d}$, the reaction yield was $91 \%$. However, the reaction time was further increased, the yield is not significantly improved, which may be related to the reaction equilibrium and the decrease of enzyme activity. Therefore, $3 \mathrm{~d}$ is chosen as the optimal reaction time.

To further explore the activity of alkaline proteinase in this novel biocatalytic protocol, a serious of $o$-aminobenzamide and $\beta$-ketoester derivatives were investigated (Table 3). A mixture of $o$-aminobenzamide $1(0.2 \mathrm{mmol})$, $\beta$-keto ester $2(0.2 \mathrm{mmol})$ and alkaline protease $(4000 \mathrm{U})$ in

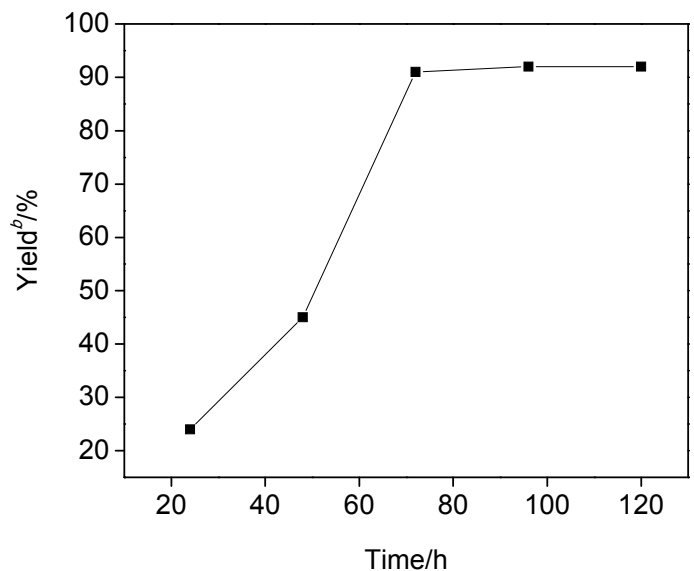

Figure 3 Effect of different reaction time on reaction yield Reaction conditions: $o$-aminobenzamide $(0.2 \mathrm{mmol})$, ethyl acetoacetate $(0.2 \mathrm{mmol})$, alkaline protease $(4000 \mathrm{U})$, ethanol $(2 \mathrm{~mL}), 50{ }^{\circ} \mathrm{C}$. Yield of isolated compound

$2 \mathrm{~mL}$ of ethanol was incubated at $50{ }^{\circ} \mathrm{C}$ and $200 \mathrm{r} / \mathrm{min}$ for $3 \mathrm{~d}$. After completion of the reaction (TLC), the mixture was concentrated under reduced pressure to afford the crude product and purified by column chromatography on silica gel (PE/EtOAc, $V: V=1: 2)$ to give the pure product 3. All substrates with various groups had good compatibility and a good to excellent yields were obtained. In addition, steric effect hardly influences the biocatalysis

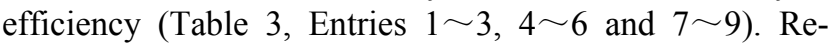
markably, the electronic effection was also not obvious. When substrates with strong electron-withdrawing groups (Table 3, Entries $4 \sim 6$ ) were applied, reactions underwent smoothly and delivered the corresponding quinazolinone derivatives in excellent yields. When the $\beta$-keto ester is acetylacetone, this reaction was observed via $\mathrm{C}-\mathrm{C}$ bond cleavage to produce 2-methyl-quinazolin-4-one (Table 3, Entry 10).

Alkaline protease, a serine-type endoprotease, is a polypeptide chain consisting of 470 amino acids, among which His-68, Asp-30 and Ser-255 constitute a catalytic triad. ${ }^{[39]}$ Combining this information and our part work, we propose a reasonable mechanism, as illustrated in Scheme 2. Initially, the proton of the amine group from $o$-aminobenzamide can be extracted by His-68 and the carbonyl group of the $\beta$-ketoester is effectively activated by Ser- 255 . Meanwhile, the amine group of $o$-aminobenzamide attacks the carbonyl group of the $\beta$-ketoester that undergoes nucleophilic addition to form the intermediate imino ester $\mathbf{A}$. Then, intermediate $\mathbf{A}$ forms the target product by dehydration which is the hydroxyl group of $\mathbf{A}$ to capture the proton of His-68, along with regeneration of alkaline protease to complete the catalytic cycle. ${ }^{[40]}$

\section{Conclusions}

In summary, alkaline protease exhibited high activity in the synthesis of quinazolinone derivatives using $o$-aminobenzamides and diverse $\beta$-keto esters. This protocol is thefirst example for synthesis of various long chain 
Table 3 Alkaline protease catalyzed synthesis of quinazolinone derivatives ${ }^{a}$

\begin{tabular}{|c|c|c|c|c|}
\hline Entry & Substrate 1 & Substrate 2 & Product 3 & Yield $^{b} / \%$ \\
\hline 1 & $1 a^{O}$ & $2 a$ & $3 a$ & 91 \\
\hline 2 & $1 a^{0}$ & $2 b$ & $3 b$ & 85 \\
\hline 3 & $1 a$ & & ${ }_{3 c}$ & 74 \\
\hline 4 & $1 b$ & $2 a$ & $3 d$ & 95 \\
\hline 5 & $1 b$ & $2 b$ & $3 e$ & 87 \\
\hline 6 & ${ }_{1 b}$ & 2c & $3 f$ & 77 \\
\hline 7 & 1c & $2 a$ & $3 g$ & 89 \\
\hline 8 & $1 c^{0}$ & $2 b$ & $3 \mathrm{~h}$ & 81 \\
\hline 9 & $1 c$ & 2c & ${ }^{\circ}{ }_{3 i}$ & 72 \\
\hline 10 & $1 a^{0}$ & $2 d$ & $\begin{array}{l}\text { O } \\
\text { 3j }\end{array}$ & 89 \\
\hline
\end{tabular}

${ }^{a}$ Reaction conditions: $o$-aminobenzamides $(0.2 \mathrm{mmol}), \beta$-ketoester $(0.2 \mathrm{mmol})$, alkaline protease $(4000 \mathrm{U})$, ethanol $(2 \mathrm{~mL}), 50{ }^{\circ} \mathrm{C}, 3 \mathrm{~d} .{ }^{b}$ Yield of isolated compound. 


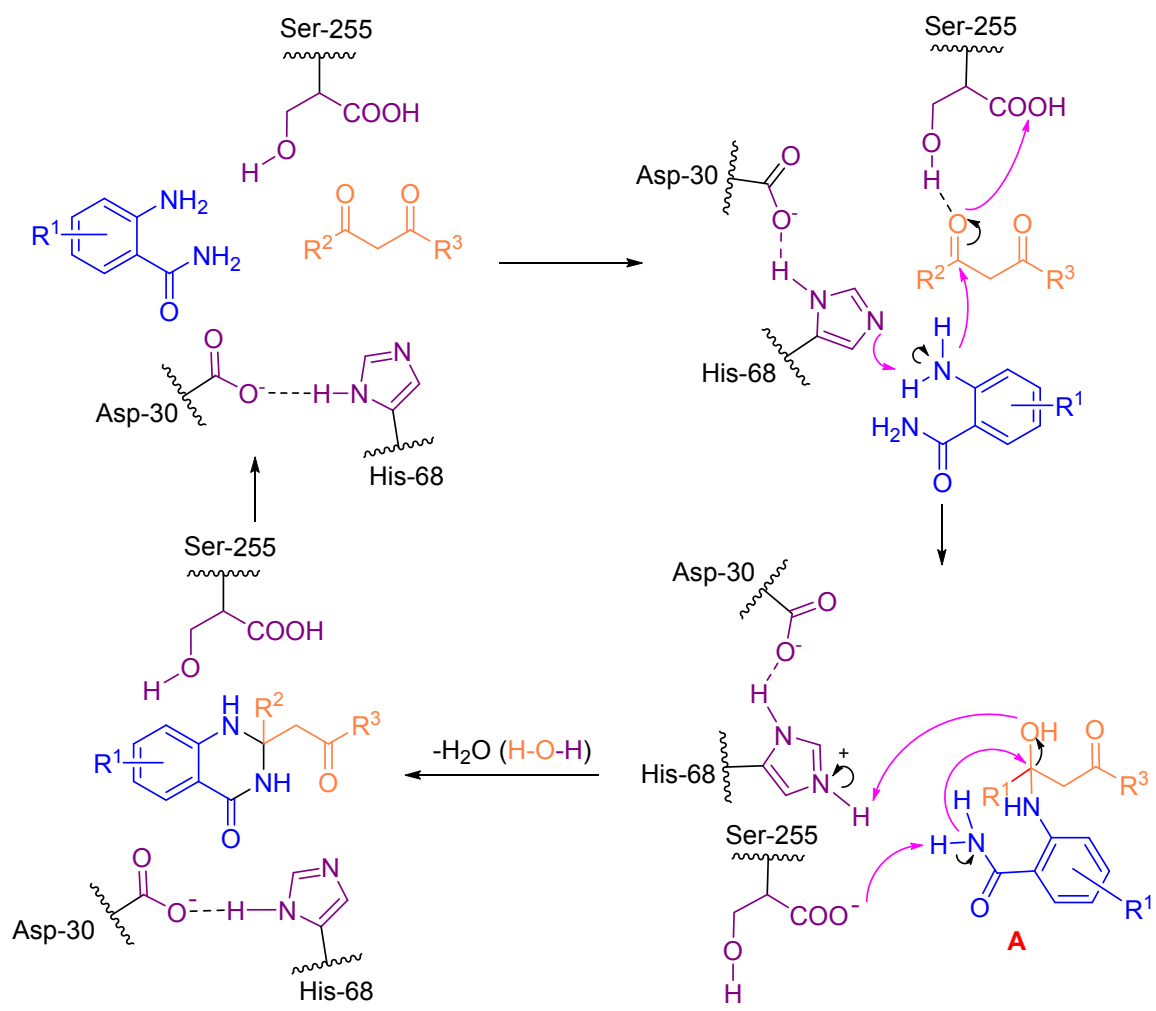

Scheme 2 Probable mechanism for synthesis of quinazolinone derivatives catalyzed by alkaline protease

quinazo linone derivatives with good functional group tolerance through an effective, eco-friendly and mild way. Further exploitation of high-activity enzymes toward non-natural reactions for the direct syntheses of structurally heterocyclic compounds is ongoing in our laboratories.

\section{Experimental section}

\subsection{Instruments and reagents}

${ }^{1} \mathrm{H}$ NMR and ${ }^{13} \mathrm{C}$ NMR spectra were recorded on a Bruker Avance III HD 600 spectrometer (Swiss Bruker, Switzerland). Mass spectrometry was measured using a LTQ-XL linear ion trap mass spectrometer (Thermo-Fisher, USA).

4.2 General procedure for alkaline protease catalyzed synthesis of quinazolinone derivatives

A mixture of $o$-aminobenzamides $1(0.2 \mathrm{mmol}), \beta$-keto ester $2(0.2 \mathrm{mmol})$ and alkaline protease $(4000 \mathrm{U}$, from Bacillus licheniformis) in $2 \mathrm{~mL}$ of ethanol was incubated at $50{ }^{\circ} \mathrm{C}$ and $200 \mathrm{r} / \mathrm{min}$ for $3 \mathrm{~d}$. After completion of the reaction (TLC), the mixture was concentrated under reduced pressure to afford the crude product and purified by column chromatography on silica gel (PE/EtOAc, $V: V=$ $1: 2)$ to give the pure product 3 .

Ethyl 2-(2-methyl-4-oxo-1,2,3,4-tetrahydroquinazolin-2yl)acetate (3a): 91\% (0.0451 g) yield. White solid, m.p. 108.2 109.1 ${ }^{\circ} \mathrm{C} ;{ }^{1} \mathrm{H}$ NMR $\left(600 \mathrm{MHz}, \mathrm{CDCl}_{3}\right) \delta: 7.84$ (dd, $J=7.8,1.5 \mathrm{~Hz}, 1 \mathrm{H}), 7.70(\mathrm{~s}, 1 \mathrm{H}), 7.28(\mathrm{ddd}, J=8.1,7.3$, $1.6 \mathrm{~Hz}, 1 \mathrm{H}), 6.82 \sim 6.78(\mathrm{~m}, 1 \mathrm{H}), 6.66 \sim 6.64(\mathrm{~m}, 1 \mathrm{H}), 5.32$ $(\mathrm{s}, 1 \mathrm{H}), 4.16 \sim 4.08(\mathrm{~m}, 2 \mathrm{H}), 2.85(\mathrm{dd}, J=163.4,15.8$ $\mathrm{Hz}, 2 \mathrm{H}), 1.63$ (s, 3H), 1.23 (t, $J=7.2 \mathrm{~Hz}, 3 \mathrm{H}) ;{ }^{13} \mathrm{C} \mathrm{NMR}$ $\left(126 \mathrm{MHz}, \mathrm{CDCl}_{3}\right) \delta: 170.66,164.02,145.71,134.20$, 128.27, 119.14, 115.04, 114.59, 68.09, 61.03, 44.58, 27.16, 14.11. ESI-HRMS calcd for $\mathrm{C}_{13} \mathrm{H}_{17} \mathrm{~N}_{2} \mathrm{O}_{3}[\mathrm{M}+\mathrm{H}]^{+}$ 249.1234, found 249.1235.

Methyl 2-(2-ethyl-4-oxo-1,2,3,4-tetrahydroquinazolin-2yl)acetate (3b): $85 \%(0.0421 \mathrm{~g})$ yield. White solid, m.p. 92.7 93.9 ${ }^{\circ} \mathrm{C} ;{ }^{1} \mathrm{H}$ NMR $\left(600 \mathrm{MHz}, \mathrm{CDCl}_{3}\right) \delta: 7.84(\mathrm{~d}$, $J=7.7 \mathrm{~Hz}, 1 \mathrm{H}), 7.37(\mathrm{~s}, 1 \mathrm{H}), 7.28(\mathrm{t}, J=7.7 \mathrm{~Hz}, 1 \mathrm{H}), 6.79$ $(\mathrm{t}, J=7.5 \mathrm{~Hz}, 1 \mathrm{H}), 6.66(\mathrm{~d}, J=8.1 \mathrm{~Hz}, 1 \mathrm{H}), 5.12(\mathrm{~s}, 1 \mathrm{H})$, 3.65 (s, 3H), 2.85 (dd, $J=156.0,15.6 \mathrm{~Hz}, 2 \mathrm{H}), 1.89$ (ddq, $J=65.7,14.6,7.4 \mathrm{~Hz}, 2 \mathrm{H}), 1.03(\mathrm{t}, J=7.4 \mathrm{~Hz}, 3 \mathrm{H}) ;{ }^{13} \mathrm{C}$ NMR $\left(126 \mathrm{MHz}, \mathrm{CDCl}_{3}\right) \delta: 170.11,163.60,145.09$, $133.16,127.07,117.62,113.83,113.06,69.87,50.86$, 42.40, 31.28, 6.85. ESI-HRMS calcd for $\mathrm{C}_{13} \mathrm{H}_{17} \mathrm{~N}_{2} \mathrm{O}_{3}[\mathrm{M}+$ $\mathrm{H}]^{+}$249.1234, found 249.1232.

Ethyl 2-(4-oxo-2-propyl-1,2,3,4-tetrahydroquinazolin-2yl)acetate (3c): $74 \%(0.0408 \mathrm{~g})$ yield, white liquid. ${ }^{1} \mathrm{H}$ NMR $\left(600 \mathrm{MHz}, \mathrm{CDCl}_{3}\right) \delta: 7.85(\mathrm{~d}, J=7.7 \mathrm{~Hz}, 1 \mathrm{H}), 7.29$ $(\mathrm{t}, J=7.7 \mathrm{~Hz}, 1 \mathrm{H}), 7.14(\mathrm{~s}, 1 \mathrm{H}), 6.80(\mathrm{t}, J=7.5 \mathrm{~Hz}, 1 \mathrm{H})$, $6.65(\mathrm{~d}, J=8.1 \mathrm{~Hz}, 1 \mathrm{H}), 5.14(\mathrm{~s}, 1 \mathrm{H}), 4.17 \sim 4.09(\mathrm{~m}, 2 \mathrm{H})$, $2.84(\mathrm{dd}, J=171.3,15.7 \mathrm{~Hz}, 2 \mathrm{H}), 1.92 \sim 1.75(\mathrm{~m}, 2 \mathrm{H})$, $1.58 \sim 1.46(\mathrm{~m}, 2 \mathrm{H}), 1.25(\mathrm{~s}, 3 \mathrm{H}), 0.94(\mathrm{t}, J=7.3 \mathrm{~Hz}, 3 \mathrm{H})$; ${ }^{13} \mathrm{C}$ NMR $\left(126 \mathrm{MHz}, \mathrm{CDCl}_{3}\right) \delta: 169.69,163.36,145.04$, $133.16,127.11,117.65,113.80,113.13,69.60,59.93$, 42.85, 40.71, 15.84, 13.07, 13.03. ESI-HRMS calcd for $\mathrm{C}_{15} \mathrm{H}_{21} \mathrm{~N}_{2} \mathrm{O}_{3}[\mathrm{M}+\mathrm{H}]^{+}$277.1547, found 277.1551.

Ethyl 2-(6-chloro-2-methyl-4-oxo-1,2,3,4-tetrahydroquinazolin-2-yl)acetate (3d): 95\% (0.0536 g) yield. Yellow solid, m.p. 161.7 162.8 ${ }^{\circ} \mathrm{C} ;{ }^{1} \mathrm{H}$ NMR (600 $\mathrm{MHz}, \mathrm{CDCl}_{3}$ ) $\delta: 7.82(\mathrm{~d}, J=2.3 \mathrm{~Hz}, 1 \mathrm{H}), 7.24(\mathrm{dd}, J=8.6,2.4 \mathrm{~Hz}, 1 \mathrm{H})$, $6.85(\mathrm{~d}, J=11.3 \mathrm{~Hz}, 1 \mathrm{H}), 6.62$ (d, $J=8.6 \mathrm{~Hz}, 1 \mathrm{H}), 5.07$ (s, 
$1 \mathrm{H}), 3.68(\mathrm{~s}, 3 \mathrm{H}), 2.83(\mathrm{dd}, J=153.9,15.8 \mathrm{~Hz}, 2 \mathrm{H})$, $1.97 \sim 1.80(\mathrm{~m}, 2 \mathrm{H}), 1.03(\mathrm{t}, J=7.4 \mathrm{~Hz}, 3 \mathrm{H}) ;{ }^{13} \mathrm{C} \mathrm{NMR}$ $\left(126 \mathrm{MHz}, \mathrm{CDCl}_{3}\right) \delta: 170.94,163.16,144.43,134.12$, $127.71,123.90,116.36,115.26,71.01,52.03,43.25,32.32$, 7.85. ESI-HRMS calcd for $\mathrm{C}_{13} \mathrm{H}_{15} \mathrm{~N}_{2} \mathrm{O}_{3} \mathrm{ClNa}[\mathrm{M}+\mathrm{Na}]^{+}$ 305.0663 , found 305.0670 .

Methyl 2-(6-chloro-2-ethyl-4-oxo-1,2,3,4-tetrahydroquinazolin-2-yl)acetate (3e): 87\% (0.0491 g) yield. Yellow solid, m.p. 164.7 164.9 ${ }^{\circ} \mathrm{C}$; ${ }^{1} \mathrm{H}$ NMR $\left(600 \mathrm{MHz}, \mathrm{CDCl}_{3}\right)$ $\delta: 7.81(\mathrm{~d}, J=2.3 \mathrm{~Hz}, 1 \mathrm{H}), 7.25(\mathrm{dd}, J=8.6,2.4 \mathrm{~Hz}, 1 \mathrm{H})$, $6.85(\mathrm{~d}, J=11.3 \mathrm{~Hz}, 1 \mathrm{H}), 6.62(\mathrm{~d}, J=8.6 \mathrm{~Hz}, 1 \mathrm{H}), 5.07$ (s, $1 \mathrm{H}), 3.68(\mathrm{~s}, 3 \mathrm{H}), 2.83(\mathrm{dd}, J=153.9,15.8 \mathrm{~Hz}, 2 \mathrm{H})$, $1.97 \sim 1.80(\mathrm{~m}, 2 \mathrm{H}), 1.03(\mathrm{t}, J=7.4 \mathrm{~Hz}, 3 \mathrm{H}) ;{ }^{13} \mathrm{C} \mathrm{NMR}$ $\left(126 \mathrm{MHz}, \mathrm{CDCl}_{3}\right) \delta: 170.94,163.16,144.43,134.12$, 127.71, 123.90, 116.36, 115.26, 71.01, 52.03, 43.25, 32.32, 7.85. ESI-HRMS calcd for $\mathrm{C}_{13} \mathrm{H}_{15} \mathrm{~N}_{2} \mathrm{O}_{3} \mathrm{ClNa}[\mathrm{M}+\mathrm{Na}]^{+}$ 305.0663, found 305.0669.

Ethyl 2-(6-chloro-4-oxo-2-propyl-1,2,3,4-tetrahydroquinazolin-2-yl)acetate (3f): $77 \%(0.0477 \mathrm{~g})$ yield. Yellow liquid. ${ }^{1} \mathrm{H} \mathrm{NMR}\left(600 \mathrm{MHz}, \mathrm{CDCl}_{3}\right) \delta: 7.81(\mathrm{~d}, J=2.4 \mathrm{~Hz}$, $1 \mathrm{H}), 7.41(\mathrm{~s}, 1 \mathrm{H}), 7.23$ (dd, $J=8.6,2.5 \mathrm{~Hz}, 1 \mathrm{H}), 6.61$ (d, $J=8.6 \mathrm{~Hz}, 1 \mathrm{H}), 5.24(\mathrm{~s}, 1 \mathrm{H}), 4.20 \sim 4.09(\mathrm{~m}, 2 \mathrm{H}), 2.82$ $(\mathrm{dd}, J=156.8,15.7 \mathrm{~Hz}, 2 \mathrm{H}), 1.91 \sim 1.74(\mathrm{~m}, 2 \mathrm{H}), 1.56 \sim$ $1.44(\mathrm{~m}, 2 \mathrm{H}), 1.26(\mathrm{t}, 3 \mathrm{H}), 0.94(\mathrm{t}, J=7.3 \mathrm{~Hz}, 3 \mathrm{H}) ;{ }^{13} \mathrm{C}$ NMR $\left(126 \mathrm{MHz}, \mathrm{CDCl}_{3}\right) \delta: 170.64,163.36,144.57$, $134.06,127.61,123.63,116.28,115.20,70.76,61.08$, 43.84, 41.73, 16.85, 14.09, 14.02. ESI-HRMS calcd for $\mathrm{C}_{15} \mathrm{H}_{20} \mathrm{~N}_{2} \mathrm{O}_{3} \mathrm{Cl}[\mathrm{M}+\mathrm{H}]^{+}$311.1157, found 311.1160.

Ethyl 2-(2,7-dimethyl-4-oxo-1,2,3,4-tetrahydroquinazolin-2-yl)acetate (3g): 89\% (0.0466 g) yield. Brown liquid. ${ }^{1} \mathrm{H}$ NMR $\left(600 \mathrm{MHz}, \mathrm{CDCl}_{3}\right) \delta: 7.73(\mathrm{~d}, J=7.9 \mathrm{~Hz}, 1 \mathrm{H})$, $7.37(\mathrm{~s}, 1 \mathrm{H}), 6.63(\mathrm{~d}, J=7.9 \mathrm{~Hz}, 1 \mathrm{H}), 6.46(\mathrm{~s}, 1 \mathrm{H}), 5.20(\mathrm{~s}$, $1 \mathrm{H}), 4.15 \sim 4.09(\mathrm{~m}, 2 \mathrm{H}), 2.83(\mathrm{dd}, J=175.7,15.8 \mathrm{~Hz}$, $2 \mathrm{H}), 2.27(\mathrm{~s}, 3 \mathrm{H}), 1.61(\mathrm{~s}, 3 \mathrm{H}), 1.24(\mathrm{t}, J=7.1 \mathrm{~Hz}, 3 \mathrm{H}) ;{ }^{13} \mathrm{C}$ NMR $\left(126 \mathrm{MHz}, \mathrm{CDCl}_{3}\right) \delta: 170.87,164.49,145.91$, $145.05,128.12,120.31,115.27,112.18,68.07,60.93$, 44.46, 27.01, 21.78, 14.10. ESI-HRMS calcd for $\mathrm{C}_{14} \mathrm{H}_{18} \mathrm{~N}_{2} \mathrm{O}_{3} \mathrm{Na}[\mathrm{M}+\mathrm{Na}]^{+}$285.1210, found 285.1213.

Methyl 2-(2-ethyl-7-methyl-4-oxo-1,2,3,4-tetrahydroquinazolin-2-yl)acetate (3h): $81 \%(0.0424$ g) yield. Brown solid, m.p. $154.5 \sim 156.4{ }^{\circ} \mathrm{C} ;{ }^{1} \mathrm{H}$ NMR $\left(600 \mathrm{MHz}, \mathrm{CDCl}_{3}\right)$ $\delta: 7.72(\mathrm{~d}, J=7.9 \mathrm{~Hz}, 1 \mathrm{H}), 7.01(\mathrm{~s}, 1 \mathrm{H}), 6.61(\mathrm{~d}, J=8.1$ $\mathrm{Hz}, 1 \mathrm{H}), 6.49 \sim 6.44(\mathrm{~m}, 1 \mathrm{H}), 5.01(\mathrm{~s}, 1 \mathrm{H}), 3.65(\mathrm{~s}, 3 \mathrm{H})$, $2.83(\mathrm{dd}, J=171.0,15.7 \mathrm{~Hz}, 2 \mathrm{H}), 2.27(\mathrm{~s}, 3 \mathrm{H}), 1.96 \sim 1.78$ $(\mathrm{m}, 2 \mathrm{H}), 1.02(\mathrm{t}, J=7.4 \mathrm{~Hz}, 3 \mathrm{H}) ;{ }^{13} \mathrm{C} \mathrm{NMR}(126 \mathrm{MHz}$, $\left.\mathrm{CDCl}_{3}\right) \delta: 171.18,164.62,146.09,145.13,128.11,120.11$, 115.12, 111.72, 70.88, 51.89, 43.30, 32.24, 21.79, 7.86. ESI-HRMS calcd for $\mathrm{C}_{14} \mathrm{H}_{18} \mathrm{~N}_{2} \mathrm{O}_{3} \mathrm{Na}[\mathrm{M}+\mathrm{Na}]^{+} 285.1210$, found 285.1211 .

Methyl 2-(7-methyl-4-oxo-2-propyl-1,2,3,4-tetrahydroquinazolin-2-yl)acetate (3i): $72 \%(0.0416 \mathrm{~g})$ yield. Brown liquid. ${ }^{1} \mathrm{H}$ NMR $\left(600 \mathrm{MHz}, \mathrm{CDCl}_{3}\right) \delta: 7.73(\mathrm{~d}, J=7.9 \mathrm{~Hz}$, $1 \mathrm{H}), 7.13(\mathrm{~s}, 1 \mathrm{H}), 6.62(\mathrm{~d}, J=7.9 \mathrm{~Hz}, 1 \mathrm{H}), 6.47(\mathrm{~s}, 1 \mathrm{H})$, $5.10(\mathrm{~s}, 1 \mathrm{H}), 4.16 \sim 4.09(\mathrm{~m}, 2 \mathrm{H}), 2.82(\mathrm{dd}, J=180.7,15.6$ $\mathrm{Hz}, 2 \mathrm{H}), 2.28(\mathrm{~s}, 3 \mathrm{H}), 1.92 \sim 1.73(\mathrm{~m}, 2 \mathrm{H}), 1.59 \sim 1.44(\mathrm{~m}$, $2 \mathrm{H}), 1.25(\mathrm{t}, J=7.1 \mathrm{~Hz}, 3 \mathrm{H}), 0.93(\mathrm{t}, J=7.3 \mathrm{~Hz}, 3 \mathrm{H}) ;{ }^{13} \mathrm{C}$ NMR (126 MHz, $\left.\mathrm{CDCl}_{3}\right) \delta: 170.81,164.53,146.12$,
$145.05,128.09,120.00,115.06,111.76,70.62,60.90$, 43.82, 41.68, 21.78, 16.85, 14.09, 14.05. ESI-HRMS calcd for $\mathrm{C}_{16} \mathrm{H}_{23} \mathrm{~N}_{2} \mathrm{O}_{3}[\mathrm{M}+\mathrm{H}]^{+}$291.1703, found 291.1705.

2-Methyl-2,3-dihydroquinazolin-4(1H)-one $\quad(\mathbf{3 j})$ : $89 \%$ $(0.0285 \mathrm{~g})$ yield. White solid, m.p. $236 \sim 238{ }^{\circ} \mathrm{C}^{[41]} ;{ }^{1} \mathrm{H}$ NMR $\left(600 \mathrm{MHz}, \mathrm{CDCl}_{3}\right) \delta: 8.27(\mathrm{~d}, J=7.9 \mathrm{~Hz}, 1 \mathrm{H})$, $7.93 \sim 7.88(\mathrm{~m}, 1 \mathrm{H}), 7.80 \sim 7.74(\mathrm{~m}, 1 \mathrm{H}), 7.68(\mathrm{~d}, J=8.1$ $\mathrm{Hz}, 1 \mathrm{H}), 7.46$ (t, $J=7.5 \mathrm{~Hz}, 1 \mathrm{H}), 7.30$ (t, $J=7.7 \mathrm{~Hz}, 1 \mathrm{H})$, $7.12(\mathrm{~s}, 1 \mathrm{H}), 6.83(\mathrm{t}, J=7.5 \mathrm{~Hz}, 1 \mathrm{H}), 6.63(\mathrm{~d}, J=8.1 \mathrm{~Hz}$, $1 \mathrm{H}), 4.22(\mathrm{~s}, 1 \mathrm{H}), 1.58(\mathrm{~s}, 6 \mathrm{H}) ;{ }^{13} \mathrm{C} \mathrm{NMR}(126 \mathrm{MHz}$, $\left.\mathrm{CDCl}_{3}\right) \delta: 164.78,164.10,153.67,149.33,145.94,134.72$, $133.98,128.31,126.89,126.26,126.21,120.42,118.76$, $114.69,114.59,67.62,29.64,22.00$.

Supporting Information ${ }^{1} \mathrm{H}$ NMR, ${ }^{13} \mathrm{C}$ NMR spectra and mass spectra for novel compounds. The Supporting Information is available free of charge via the internet at http://sioc-journal.cn/.

\section{References}

[1] Yan, B.-R.; Lv, X.-Y.; Du, H.; Bao, X.-P. Chin. J. Org. Chem. 2016, 36, 207 (in Chinese). (闵柏任, 吕新阳, 杜欢, 鲍小平, 有机化学, 2016, 36, 207.)

[2] Abdolmohammadi, S.; Karimpour, S. Chin. Chem. Lett. 2016, 27, 114.

[3] Ghosh, S. K.; Nagarajan, R. RSC Adv. 2016, 6, 27378.

[4] Shiri, L.; Narimani, H.; Kazemi, M. Appl. Organomet. Chem. 2018, 32, e3999.

[5] Ghorbani-Choghamarani, A.; Darvishnejad, Z.; Norouzi, M. Appl. Organomet. Chem. 2015, 29, 707.

[6] Norouzi, M.; Ghorbani-Choghamarani, A.; Nikoorazm, M. RSC Adv. 2016, 6, 92387.

[7] Abdollahi-Alibeik, M.; Shabani, E. Chin. Chem. Lett. 2011, 22, 1163.

[8] Khan Abdul, A.; Mitra, K.; Mandal, A.; Baildya, N.; Mondal Mohabul, A. Heteroat. Chem. 2017, 28, e21379.

[9] Das Gupta, A.; Samanta, S.; Mallik, A. K. Org. Prep. Proced. Int. 2015, 47, 356.

[10] Shiri, L.; Ghorbani-Choghamarani, A.; Kazemi, M. Appl. Organomet. Chem. 2016, 31, e3596.

[11] Nikoorazm, M.; Ghorbani-Choghamarani, A.; Khanmoradi, M. RSC Adv. 2016, 6, 56549.

[12] Katla, R.; Chowrasia, R.; da Silva, C. D. G.; de Oliveira, A. R.; dos Santos, B. F.; Domingues, N. L. C. Synthesis 2017, 49, 5143.

[13] Rajaka, L.; Penumati, N. R.; Nagaiah, K.; Poornachandra, Y.; Kumar, C. G. Synth. Commun. 2015, 45, 1893.

[14] Mikls, F.; Hum, V.; Fülöp, F. ARKIVOC 2014, 6, 25.

[15] Tamaddon, F.; KazemiVarnamkhasti, M. Carbohydr. Res. 2017, 437, 9.

[16] Borase, P. N.; Thale, P. B.; Shankarling, G. S. RSC Adv. 2016, 6, 63078 .

[17] Tamaddon, F.; Pouramini, F. Synlett 2014, 25, 1127.

[18] Nguyen, T. B.; Ermolenko, L.; Al-Mourabit, A. Green Chem. 2013, $15,2713$.

[19] Watson, A. J. A.; Maxwell, A. C.; Williams, J. M. J. Org. Biomol. Chem. 2012, 10, 240.

[20] Gao, L.; Ji, H.; Rong, L.; Tang, D.; Zha, Y.; Shi, Y.; Tu, S. J. Heterocycl. Chem. 2011, 48, 957.

[21] Cerqueira Pereira, S.; Bussamara, R.; Marin, G.; Lima Camargo Giordano, R.; Dupont, J.; de Campos Giordano, R. Green Chem. 2012, 14, 3146.

[22] Kloosterman, W. M. J.; Roest, S.; Priatna, S. R.; Stavila, E.; Loos, K. Green Chem. 2014, 16, 1837.

[23] Paggiola, G.; Hunt, A. J.; McElroy, C. R.; Sherwood, J.; Clark, J. H. Green Chem. 2014, 16, 2107. 
[24] Yang, M.; Luo, M.; Xian, X. J. Chongqing Univ. (Engl. Ed.) 2011, $10,23$.

[25] O'Brien, P. J.; Herschlag, D. Chem. Biol. 1999, 6, R91-R105.

[26] Acharya, C.; Mandal, M.; Dutta, T.; Ghosh, A. K.; Jaisankar, P. Tetrahedron Lett. 2016, 57, 4382.

[27] Li, R.; Li, Z.-L.; Zhou, H.-Y.; He, Y.-H.; Guan, Z. J. Mol. Catal. B: Enzym. 2016, 126, 90.

[28] Cai, J.-F.; Guan, Z.; He, Y.-H. J. Mol. Catal. B: Enzym. 2011, 68, 240.

[29] Zandvoort, E.; Geertsema Edzard, M.; Baas, B. J.; Quax Wim, J.; Poelarends Gerrit, J. Angew. Chem. 2012, 124, 1266.

[30] Zhang, M.-J.; Li, R.; He, Y.-H.; Guan, Z. Catal. Commun. 2017, 98, 85.

[31] Ding, X.; Zhang, X.-D.; Dong, C.-L.; Guan, Z.; He, Y.-H. Catal. Lett. 2018, 148, 757 .

[32] He, T.; Li, K.; Wu, M.-Y.; Feng, X.-W.; Wang, N.; Wang, H.-Y.; Li, C.; Yu, X.-Q. J. Mol. Catal. B: Enzym. 2010, 67, 189.

[33] Guan, Z.; Song, J.; Xue, Y.; Yang, D.-C.; He, Y.-H. J. Mol. Catal. B: Enzym. 2015, 111, 16.
[34] Tian, X.; Zheng, L. 2015 China Enzyme Engineering and Sugar Bioengineering Symposium Abstract Collection 2015.

[35] Acharya, C.; Achari, A.; Jaisankar, P. Tetrahedron Lett. 2018, 59, 663.

[36] Sun, D.-Z.; Jiang, G.-F.; Xie, Z.-B.; Le, Z.-G. Chin. J. Chem. 2015, 33, 409.

[37] Le, Z.-G.; Liang, M.; Chen, Z.-S.; Zhang, S.-H.; Xie, Z.-B. Molecules 2017, 22, 762 .

[38] Xie, Z.-B.; Zhang, S.-G.; Jiang, G.-F.; Liang, M.; Le, Z.-G. Chin. J. Org. Chem. 2017, 37, 514 (in Chinese). (谢宗波, 张士国, 姜国芳, 梁萌, 乐长高, 有机化学, 2017, 37, 514.)

[39] Yang, N.; Nan, J.; Broströmer, E.; Hatti-Kaul, R.; Su, X. Proteins 2010, 73, 1072.

[40] Liu, L.-S.; Xie, Z.-B.; Zhang, C.; Fu, L.-H.; Zhu, H.-B.; Le, Z.-G. Green Chem. Lett. Rev. 2018, 11, 503.

[41] Li, Z.-W.; Dong, J.-Y.; Chen, X.-L.; Li, Q.; Zhou, Y.-B.; Yin, S.-F. J. Org. Chem. 2015, 80, 9392. 